\title{
Histopathology in the Diagnosis and Classification of Acute Myeloid Leukemia, Myelodysplastic Syndromes, and Myelodysplastic/Myeloproliferative Diseases
}

\author{
Attilio Orazi \\ Indiana University School of Medicine, Division of Hematopathology, Indianapolis, Ind., USA
}

\section{Key Words \\ Histopathology • Acute myeloid leukemia • \\ Myelodysplastic syndromes $\cdot$ Myeloproliferative disorders}

\begin{abstract}
In spite of the impressive advances in the area of molecular pathology, bone marrow morphology remains the diagnosis cornerstone to identify the various subtypes of myeloid neoplasms. Morphological examination of the bone marrow requires both bone marrow aspirate and bone marrow trephine biopsy. Immunohistochemistry of bone marrow biopsy with markers reactive in paraffin-embedded tissues represents a powerful diagnostic tool; its results can be easily correlated with those obtained by other techniques such as flow cytometry and genetic analysis, and above all, the clinical findings. The role of the bone marrow biopsy will be particularly stressed in this review article. Particular emphasis is being given to the correct identification of cases of myeloid neoplasms associated with myelofibrosis and for which the bone marrow biopsy represents the only available diagnostic mean. Moreover, the often low cellular yield of the bone marrow aspirate in these cases may also be insufficient to obtain adequate cytogenetic information. Such cases include two subtypes of acute myeloid leukemia which typically cause diagnostic difficulties: acute megakaryoblastic leukemia and acute panmyelosis with myelofibrosis (acute myelosclerosis). Acute myeloid leukemia with multilineage
\end{abstract}

dysplasia, therapy-related myelodysplastic syndrome/therapy-related acute myeloid leukemia and de novo myelodysplastic syndromes (MDS) will also be discussed. The value of bone marrow biopsy in this group of disorders is generally well established. In MDS, in particular, bone marrow biopsy may help in confirming a suspected diagnosis by excluding reactive conditions in which dyshematopoietic changes may also be observed. It can increase the diagnostic accuracy and helps in refining the IPPS risk evaluation system. Among the alterations detected by bone marrow biopsy, a prognostically important finding is the presence of aggregates or clusters of immature myeloid precursor cells (myeloblasts and promyelocytes). These can also be identified by immunohistochemistry with CD34, an antigen expressed in progenitor and early precursor marrow cells, which can be used to demonstrate pathological accumulations of blasts in aggressive subtypes of myeloid neoplasms. Immunohistologic analysis is especially helpful in cases of MDS with fibrosis and cases with hypocellular marrows (hypoplastic MDS). In both of these variants, the presence of reticulin fibrosis or fatty changes in the bone marrow can make accurate disease characterization very difficult or impossible using bone marrow aspirates. Finally, the important group of the myelodysplastic/myeloproliferative disorders can only be accurately categorized by a careful multiparametric approach in which the bone marrow biopsy exerts a pivotal role.

Copyright $\odot 2007$ S. Karger AG, Basel

\section{KARGER}

Fax +4161306 1234

E-Mail karger@karger.ch

www.karger.com
(C) 2007 S. Karger AG, Basel

$1015-2008 / 07 / 0742-0097 \$ 23.50 / 0$

Accessible online at:

www.karger.com/pat
Attilio Orazi, Director Division of Hematopathology

Indiana University School of Medicine, Clarian Pathology Laboratory

Room 5000D/3, Indianapolis, IN 46202-5200 (USA)

Tel. +1 317491 6510, Fax +1 3174916114

E-Mail aorazi@iupui.edu or rdbennet@iupui.edu 
Table 1. Bone marrow aspirate and biopsy

Bone marrow aspirate

Blast cell count

$\mathrm{M}:$ E ratio and detailed myelogram

Cytochemistry

Dysplasia

Flow cytometry

Cytogenetics/molecular genetic techniques

Bone marrow biopsy

Cellularity, lineage prevalence, architectural disturbances

Fibrosis and stromal changes

Immunohistochemistry

Dysmegakaryopoiesis

True blast cell count

Microdissection-based approaches

Table 2. AML classification according to WHO 2001

AML with specific cytogenetic/genetic abnormalities

[ $\mathrm{t}(8 ; 21), \mathrm{t}(15 ; 17)$, inv or $\mathrm{t}(16), 11 \mathrm{q} 23]$

AML-NOS [7 FAB types (M0-M7) plus basophilic and APMF]

AML with multilineage dysplasia ( \pm prior MDS)

t-AML/MDS (alkylating, topoisomerase)

In the universally adopted WHO 2001 classification system [1], disease entities are defined by a combination of morphology, immunophenotype, genetics, and clinical features. The relative importance of each of these features varies among diseases - no gold standard 'one size fits all' is established. Neoplasms are stratified primarily according to their lineage (e.g. myeloid, lymphoid, mast cell, histiocytic). The diagnosis follows a stepwise approach: first, in establishing a hematopoietic neoplasm versus other non-hematopoietic neoplastic diseases and reactive conditions; second, in identifying the main type of hematopoietic neoplasm, e.g. acute myeloid leukemia (AML) versus acute lymphoblastic leukemia (ALL), AML versus myelodysplastic syndromes (MDSs), and chronic myeloproliferative disorders (CMPDs) versus myelodysplastic/myeloproliferative (MDS/MPD) diseases; third, in identifying the specific WHO subtypes of a given hematopoietic neoplasm, e.g. AML, monoblastic versus megakaryoblastic, MDS, refractory anemia (RA) versus refractory anemia with excess of blasts (RAEB), and MDS/MPD, chronic myelomonocytic leukemia (CMML) versus atypical chronic myeloid leukemia (aCML).
Table 3. Immunohistological reagents useful in separating AML from ALL in paraffin sections

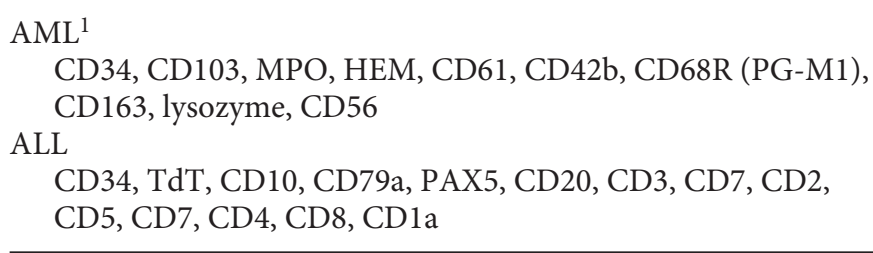

$\mathrm{AML}=$ Acute myeloid leukemia; $\mathrm{ALL}=$ acute lymphoblastic leukemia; HEM = hemoglobin; MPO = myeloperoxidase.

${ }^{1}$ AML with specific cytogenetic abnormalities PAX5, CD79a $[\mathrm{t}(8 ; 21)] ; \mathrm{NPM}$ cyto+ (normal karyotype).

Table 4. Paraffin immunophenotype: limited correlation with FAB subtypes

\begin{tabular}{ll}
\hline M0 & CD34+; MPO+/-; CD68 (KP1)+/-; CD68 (PG-M1)- \\
M1/M2 & CD34+/-; MPO+; CD68 (KP1)+; CD68 (PG-M1)- \\
M3 & MPX++; HLA-DR-; CD34-; CD68 (PG-M1)- \\
M4 & MPX+; CD68 (PG-M1)+/-; CD34- \\
M5 & CD68 (PG-M1)+; MPO+/-; CD34+/- \\
M6 & HB, Glyco A+ cells >50\%; MPO+/-; CD34+/- \\
M7 & CD61 >50\% blasts; MPO-; CD34+/- \\
\hline
\end{tabular}

The separation of the various entities belonging to the group of myeloid neoplasms is best achieved by a multiparametric approach. However, the bone marrow morphology retains its importance in reaching a correct diagnosis. Additionally, it might be the only approach available in many countries and the one upon which the practicing hematologist has to rely. Morphology supplemented by immunohistochemistry with markers reactive in routinely processed tissues represents a powerful diagnostic tool on its own. Its results can be usually easily correlated to those obtained with other non-morphologic techniques such as flow cytometry and genetic analysis, and above all the clinical findings.

Morphological examination of the bone marrow requires both a properly prepared bone marrow aspirate smear and bone marrow trephine biopsy. The latter should be of adequate length and carefully processed. The two approaches effectively complement each other, as can be seen in table 1 . The role of the bone marrow biopsy will be particularly stressed in this presentation. 
Table 5. AML with fibrosis

Acute megakaryoblastic

Acute panmyelosis with myelofibrosis

Other AML-NOS (rare)

Acute leukemia with multilineage dysplasia de novo or secondary to MDS, MDS/MPD or CMPD

Therapy-related (alkylating agents)

Table 6. Acute megakaryoblastic leukemia (AML-M7)

5\% ANLL, all ages

Associations: Down's syndrome, MNSGCTs ${ }^{1}$

Secondary to CMPD or MDS

Hepatosplenomegaly, lytic lesions

PB: Pancytopenia, blasts, no dacrocytosis

BM: Blasts $\geq 20 \%$; $\geq 50 \%$ of blasts megakaryocytic lineage,

fibrosis $(+3)$

Cytogenetics: variable, inv $3, \mathrm{t}(1 ; 22), \mathrm{i}(12 \mathrm{p})$

GATA1 somatic mutations (expression of GATA1s)

\footnotetext{
${ }^{1}$ Mediastinal non-seminomatous germ cell tumors [Orazi et al., Cancer 1994].
}

\section{Acute Myeloid Leukemia}

The diagnosis and classification of acute leukemia currently requires a combination of morphology, cytochemistry, immunophenotyping and cytogenetics (or molecular genetics) for complete diagnosis of many of the disease types. The main AML subtypes including the three subtypes identified on the basis of a specific cytogenetic abnormality are listed in table 2 .

Although the gold standard in diagnosing AML is a combination of bone marrow aspirate smear morphology, cytochemistry, flow cytometry, and cytogenetics, the bone marrow biopsy yields useful information which can be crucially important in several diagnostic settings and is therefore a mandatory step. What is the role of the bone marrow biopsy in the initial disease assessment? First, it is impossible to be sure 'upfront' of the adequacy of the bone marrow aspirate. In fibrotic or fatty marrows only the biopsy can provide for a correct assessment of the blast count. Additionally, given the importance of followup (e.g. post-chemotherapy and/or bone marrow transplant) bone marrows, it is necessary to create a baseline 'picture' against which to compare them. This can greatly help in excluding the presence of a residual minimal leukemic disease.

Histopathology in the Diagnosis and Classification of Myeloid Neoplasms
Table 7. Antibodies which are known to recognize abnormal MK forms and MK blasts

\begin{tabular}{lll}
\hline & MKs/atypical MKs & MK blasts \\
\hline vWF & good & inadequate \\
CD42b & good & good \\
CD61 (processing-dependent) & good & good \\
CD31 (nonspecific) & good & good \\
\hline
\end{tabular}

Table 8. Acute panmyelosis with myelofibrosis (acute onset, no splenomegaly, rapidly fatal)

PB Pancytopenia, no teardrops, non-leukemic
BM Proliferative disorder of all three cell lines:
Small-size MKs with disperse chromatin,
non- or hypolobulated nuclei
Blasts variable increased (10-25\%)
No or rare MK blasts
Degree of fibrosis is variable, but usually 3+

In all cases, the diagnostic value of the bone marrow biopsy can be greatly enhanced by adding immunohistochemistry. The latter technique employs antibodies similar or comparable to those traditionally used by flow cytometry and immunofluorescence. Some of the antibodies reactive with paraffin-embedded bioptic tissue which can be useful in distinguishing AML from ALL are found listed in table 3 . In the large group of the AML, the AML not otherwise specified (AML-NOS includes the 6 FAB classification-derived subtypes (M0M6) plus acute megakaryoblastic leukemia, acute basophilic leukemia, and acute panmyelosis with myelofibrosis) [2] (table 2), immunohistochemistry may be helpful in the distinction between the various AML subtypes (table 4 ).

Cases of AML associated with marrow fibrosis represent a diagnostic challenge (fig. 1). The fibrotic variants of AML are summarized in table 5. Among the fibrotic AML, two subtypes which typically cause the greatest diagnostic difficulties are acute megakaryoblastic leukemia (AMKL) and acute panmyelosis with myelofibrosis (APMF; acute myelosclerosis). 
1

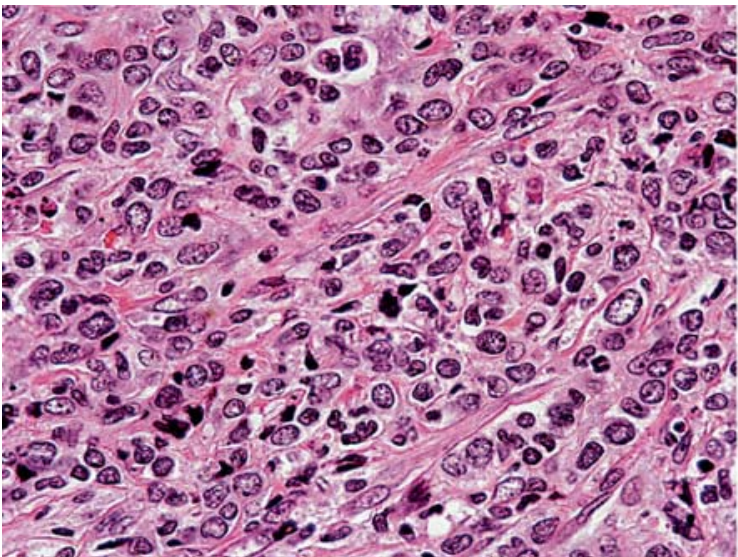

2

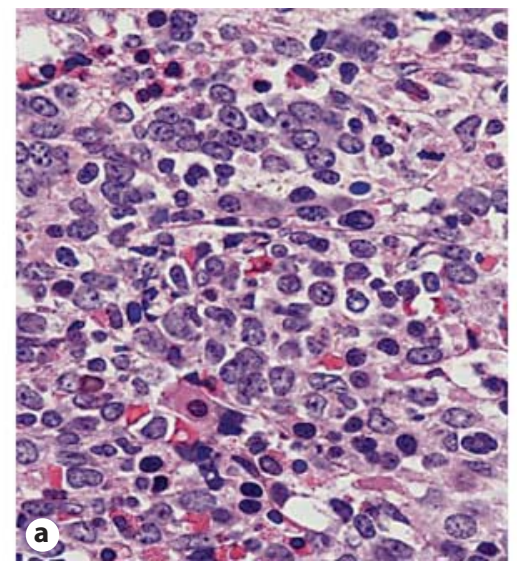

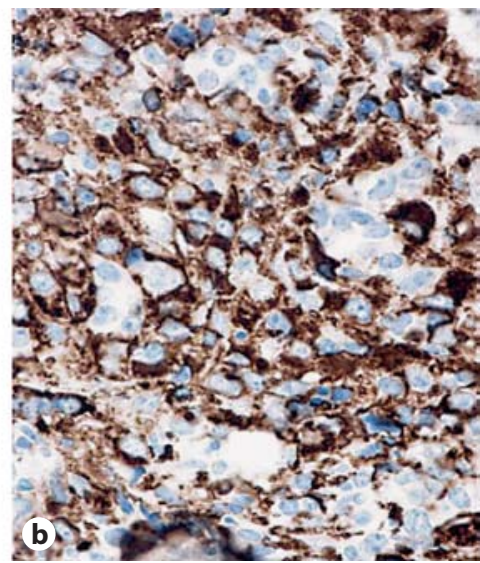

3
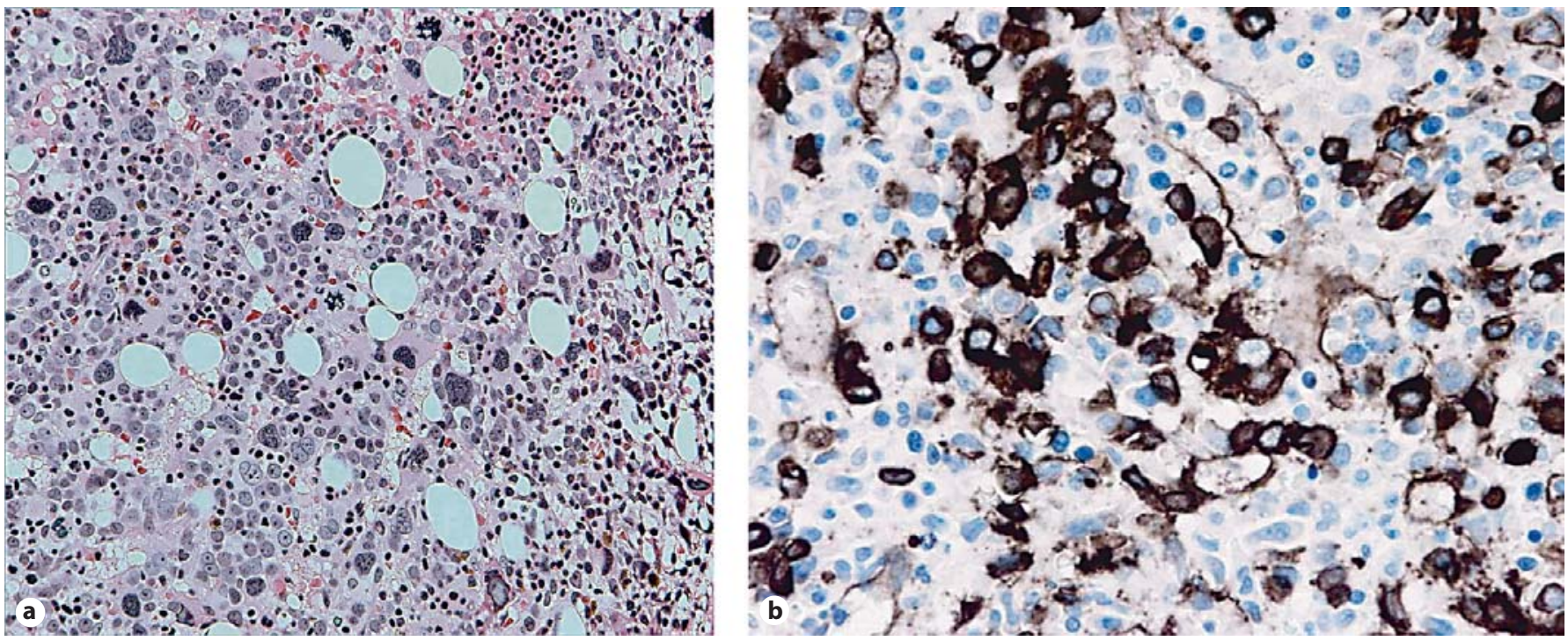

Fig. 1. AML-NOS with fibrosis. Dry tap aspirate. The myeloid nature of the blasts in this case can only be confirmed by immunostaining. Fig. 2. AMKL. a AMKL, poorly differentiated example. Note the large cell lymphoma-like morphology of the blasts. b CD42 confirms their megakaryoblastic nature.

Fig. 3. APMF. a Panmyelotic proliferation with many atypical megakaryocytes. b CD34 confirms the presence of an increased number of blasts. c CD42 staining highlights the dysplastic megakaryocytes. Note the absence of megakaryoblasts.

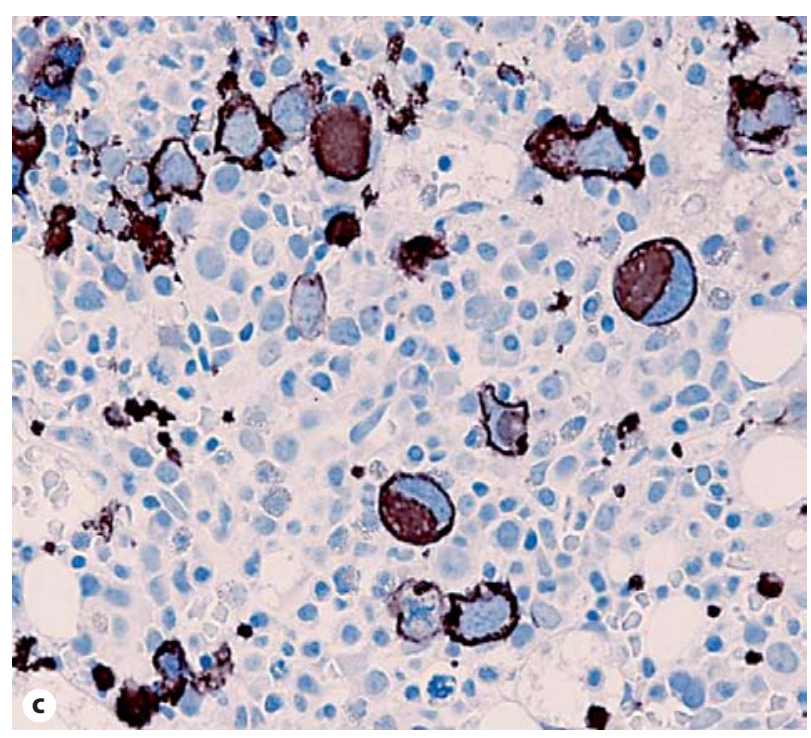




\section{Acute Megakaryoblastic Leukemia}

De novo AMKL is an uncommon disease representing $1 \%$ or less of AMLs [1]. The pediatric forms include cases associated with Down's syndrome, often following resolution of a transient MPD, occurring at around 2 years of age, and cases associated with $\mathrm{t}(1 ; 22)(\mathrm{p} 13 ; \mathrm{q} 13)$, which usually occurs as a sole abnormality in the first 6 months of life $[3,4]$. In adult males, an association between AMKL and mediastinal non-seminomatous germ cell tumors has been reported [5]. The main features of AMKL are summarized in table 6. AMKL is characterized by a relatively uniform blast cell proliferation, often 'lymphoid'looking cells with varying amounts of basophilic cytoplasm, and without Auer rods (fig. 2a). Cytoplasmic blebs may be present, but are not specific enough for use as an absolute diagnostic criterion. The blast cells are myeloperoxidase and Sudan black B negative, but may be positive for the non-specific esterase such as $\alpha$-naphthyl acetate esterase (the blasts are sodium-fluoride sensitive though). By flow cytometry, the blasts may express myeloid-associated antigens, but should also express at least two megakaryocyte-associated antigens, such as CD41, CD42, and/or CD61. In AMKL, however, bone marrow aspiration is frequently unsuccessful due to marrow fibrosis. In these cases, cytochemistry and flow cytometry can be performed using peripheral blood samples, since circulating blasts are present in the majority of the cases. In most cases, however, bone marrow biopsy supplemented by immunohistology (table 7) may be required for appropriate characterization [6]. CD42 and CD61 antibodies are used to confirm the megakaryoblastic nature of the cells in tissue sections. Von Willebrand factor (vWF; factor VIII) can also be used but is less frequently positive. The prognosis for AMKL is poor.

\section{Acute Panmyelosis with Myelofibrosis}

APMF is a rare subtype of AMLs and corresponds also to $<1 \%$ of cases [1]. The WHO criteria for the diagnosis of APMF include: panmyelosis, significant marrow fibrosis, pancytopenia, normal erythrocyte morphology, lack of splenomegaly, and a rapidly fatal course (table 8). Bone marrow aspiration is usually unsuccessful and the bone marrow biopsy supplemented with immunohistology is required for establishing this diagnosis $[6,7]$. The bone marrow biopsy (fig. 3a) shows a hypercellular, diffusely fibrotic bone marrow with an increased number of multilineage immature hematopoietic elements, and

Histopathology in the Diagnosis and Classification of Myeloid Neoplasms conspicuously dysplastic megakaryocytes predominately of small size showing variable degrees of atypia, including the presence of hypolobulated or non-lobulated nuclei with dispersed chromatin. Foci of blasts are found scattered throughout the marrow $[6,7]$. The overall frequency of blasts in APMF marrows is uncertain. In a recent study based on bone marrow biopsy, we found a blast frequency comprised between 10 and $25 \%$ with a median value of $22.5 \%$ [6], however its precise determination is not considered a diagnostic requirement, according to the WHO system. Most of the blasts express CD34 (fig. 3b) and are negative for the megakaryocyte-associated markers such as CD42 (fig. 3c) and CD61 [6]. The course is rapidly fatal, often terminating with an overtly leukemic phase. In the terminal stage, splenomegaly may be observed, which usually results from leukemic infiltration of the red pulp. The disease usually occurs in adults, but rare cases have been described in children. The main differential diagnosis is MDS with fibrosis (MDS- $\mathrm{f}$ ) associated with an excess of blasts and cases of AML with multilineage dysplasia with a low blast frequency, and AMKL with a 'dry tap' and no circulating blasts. Distinction, in some of these cases, may however be arbitrary and clinically irrelevant.

\section{Acute Myeloid Leukemia with Multilineage Dysplasia and Therapy-Related Acute Myeloid Leukemia}

AML with multilineage dysplasia (AML-MLD) may occur de novo, may follow a known MDS, or be therapyrelated (TR-AML) $[1,8,9]$. This group of diseases shares an increased frequency of complex cytogenetic abnormalities, deletions or chromosomes 5 or 7 , trisomies, or abnormalities of chromosome band $3 \mathrm{q} 21$, and a poor prognosis. AML-MLD and TR-AML are diseases that usually show dysplastic changes in the non-blast marrow and peripheral blood elements. The WHO classification defines AML-MLD as $20 \%$ or more bone marrow or peripheral blood blast cells with $50 \%$ or more dysplastic cells in at least two cells lines (erythroid, granulocytic and/or megakaryocytic) [9] (table 9). Dysplastic changes may be seen in both peripheral blood and bone marrow samples. Red blood cell changes include anisopoikilocytosis of peripheral blood red cells, including hypochromic teardrop-shaped cells and macrocytes, dimorphic red cell populations of the blood, nuclear-cytoplasmic asynchrony of red cell precursors, megaloblastic changes and irregularities of red cell precursor nuclei. Multinu- 
Table 9. Acute leukemia with multilineage dysplasia

Neoplastic process predominately composed of one cell type (myeloblast)

Dysplasia in $\geq 50 \%$ cells in $\geq 2$ cell lines

Absence/rarity of megakaryoblasts

Lesser degrees of fibrosis tures and may or may not have associated multilineage dysplasia. Abnormalities of the MLL gene of 11q23 and AML1 gene of 21q22 are commonly present in these cases, but a variety of other abnormalities including $\mathrm{t}(8 ; 21)$, $\mathrm{t}(3 ; 21), \operatorname{inv}(16), \mathrm{t}(8 ; 16), \mathrm{t}(15 ; 17)$ or $\mathrm{t}(9 ; 22)$ may also be seen [13]. TR-AML is also generally associated with a poor prognosis.

\section{Biopsy Facilitates the Distinction between AML and MDS}

The bone marrow biopsy can be used to provide correct blasts enumeration (see next section on MDS) and to avoid 'undercounts' in the aspirate due to spotty cellularity, fatty marrows, or fibrosis. Discrepancy between bone marrow aspirate and bone marrow biopsy: the highest blast count result should be used. Blasts are more often in clusters (3-5 cells) in aggressive MDS subtypes, RAEB in particular (fig. 4). Conversely, in AML the blasts usually are arranged in aggregates ( $>5$ cells) or in confluent sheets. In hypoplastic AML (fig. 5a), the blasts are often interstitially located with an Indian file appearance. Their visibility can be enhanced by immunohistology (fig. 5b).

Immunohistology can also be helpful in drawing the line between RAEB and AML by facilitating the detection of the blasts and the assessment of their distribution. For cases rich in megaloblastoid erythroblasts, immunohistology for glycophorin or antihemoglobin may be helpful in distinguishing those cells from the myeloblasts (e.g. in cases of RAEB or AML-M6).

\section{Myelodysplastic Syndromes}

The diagnostic work-up of MDS requires morphologic evaluations of peripheral blood, marrow aspirate, and bone marrow biopsy, in the light of complete blood count results and adequate clinical information. Correlation with marrow cytogenetics is always recommended. It needs to be stressed, however, that the presence of a 'negative' karyotype does by no means exclude a diagnosis of MDS. To increase the yield of cytogenetic or genetic anomalies, FISH analysis as well as other less frequently available techniques (e.g. loss of heterozygosity for tumor suppressor genes, spectral karyotyping) can also be successful in demonstrating clonal hematopoiesis in cases of MDS. ase II inhibitor-related disease generally has a shorter latency period of 2-3 years, tends to have monocytic fea- 


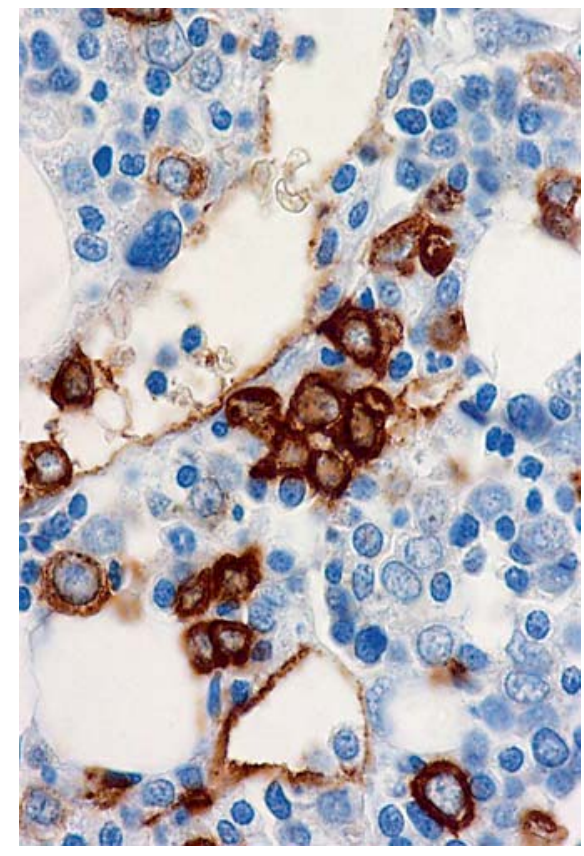

Fig. 4. MDS-RAEB. CD34 demonstrates the presence of clusters of blasts, a characteristic finding in aggressive subtypes of MDS.
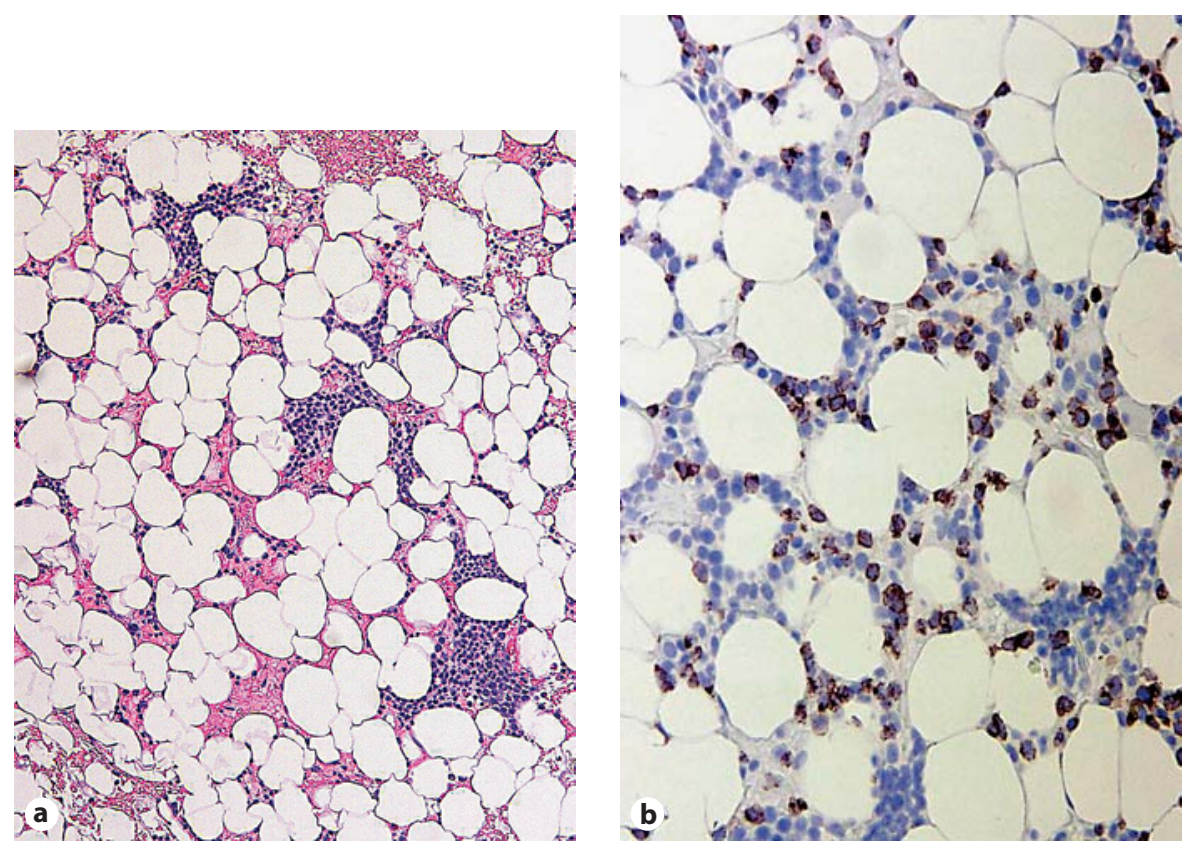

Fig. 5. a Hypoplastic AML. It is difficult to appreciate the high number of blasts located interstitially among the fat lobules. b CD34 facilitates their identification (note that not all AMLexpress CD34).

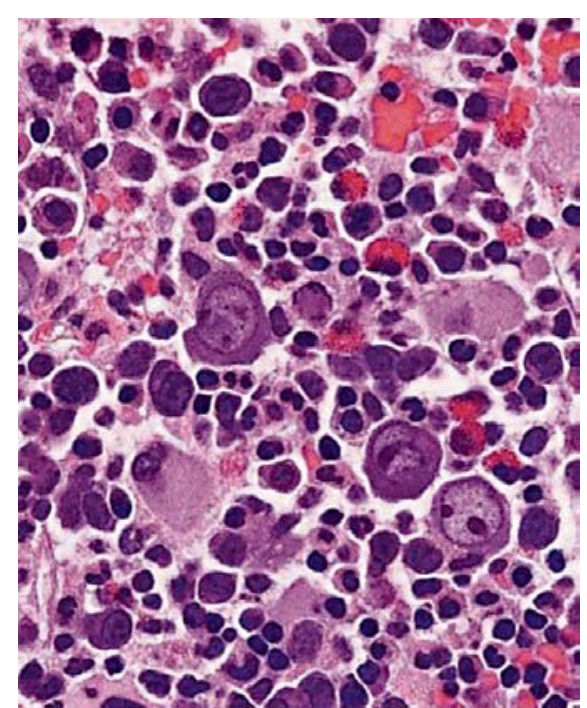

Fig. 6. Chronic parvovirus infection. Note the abnormal looking appearance of erythroblasts which can be mistaken for evidence of severe dyserythropoiesis.
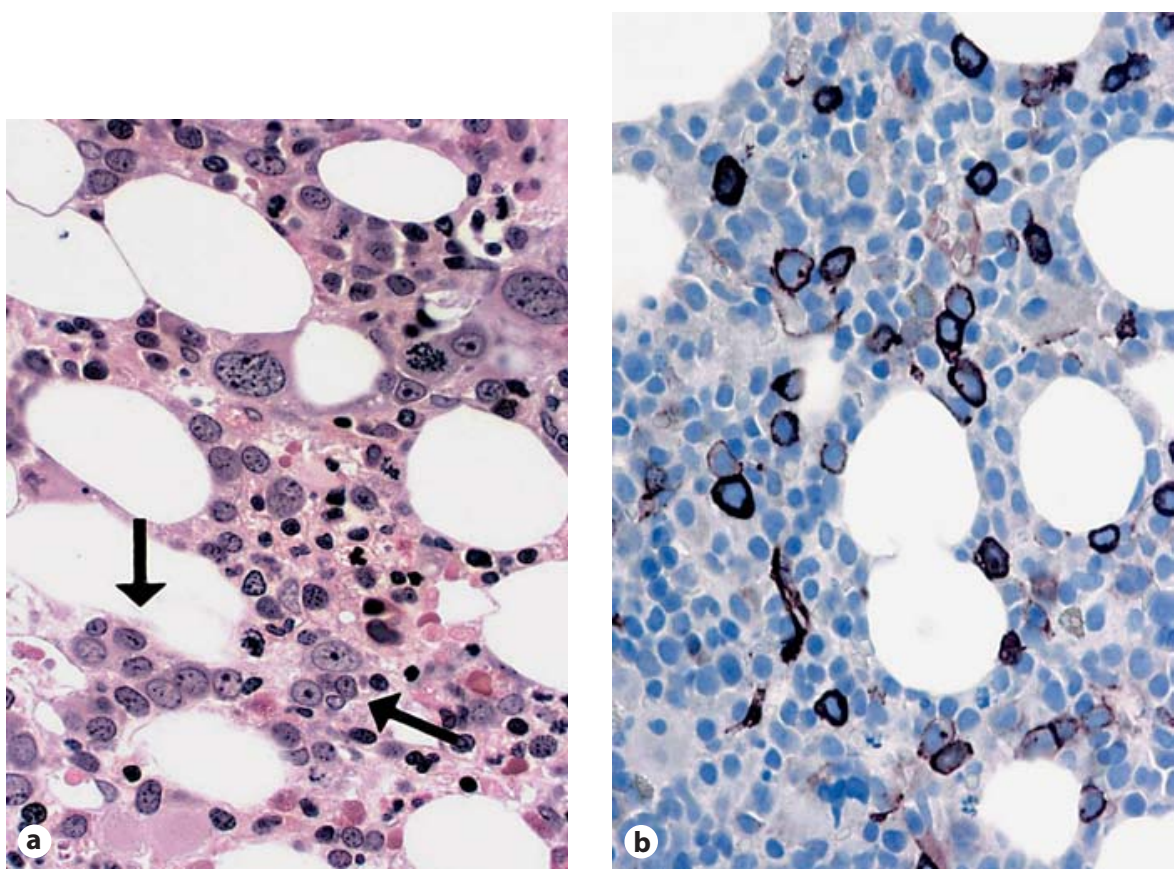

Fig. 7. MDS-RAEB. a ALIP (arrows) as seen in an HE-stained section of bone marrow biopsy. $\mathbf{b}$ CD34 staining confirms the presence of an increased number of blasts. 
Table 11. Dyshematopoietic changes MDS-like (non-clonal)

\begin{tabular}{lll}
\hline Dyserythropoiesis: non-MDS & Dysgranulopoiesis: non-MDS & Dysmegakaryopoiesis: non-MDS \\
\hline $\mathrm{B}_{12} /$ folate deficiencies & Chemotherapy/bone marrow regeneration & Infections (HIV) \\
Methotrexate and other chemotherapy & $\mathrm{B}_{12} /$ folate deficiency & Chemotherapy \\
Alcohol and other toxic compounds & CSFs treatment & Paraneoplastic myelofibrosis \\
Autoimmune conditions & Infections (HIV, $\mathrm{B}_{19}$ ) & Autoimmune myelofibrosis \\
Aplastic anemia/PNH & Malignancy-associated (paraneoplastic) & Status post-transplant \\
Transplant recipients & Congenital (Fanconi anemia) & TMPD (Down syndrome) \\
Infections (parvovirus, HIV) & Drug (purine analog) & \\
Hemophagocytic lymphohistiocytosis & & \\
Congenital dyserythropoietic anemias & & \\
\hline
\end{tabular}

Table 12. Pseudo-ALIPs: immunohistology

\begin{tabular}{ll}
\hline Erythroblasts (megaloblastic anemia) & HB (glycophorin A)+ \\
Promyelocytes (after G-CSF) & MPO+/CD34-/CD117- \\
Monocytes & CD68 (PG-M1)+ \\
$\quad$ Large-sized lymphoid cells & Lymphoid markers+ \\
\hline
\end{tabular}

Table 13. MDS with fibrosis ( $\geq 2$ reticulin fibrosis) [see 37]

$10-15 \%$ p-MDS

RAEB $>>$ other types

No organomegaly

Increased MKs and dysmegakaryopoiesis

Clusters of CD34

No or rare MK-blasts
The previously used FAB classification [2] was based entirely on findings identifiable by cytological analysis of stained smears of peripheral blood and marrow aspirate. The main criteria for subdividing MDS were the percentage of blasts in the peripheral blood and in bone marrow aspirates, and the identification of dysplastic changes in at least one of the three main marrow cell lines. The new and more comprehensive approach used by the WHO system stresses the importance of integrating other techniques such as bone marrow biopsy histology, cytogenetics, and molecular genetics, which may provide clinically relevant information. However, not all of these techniques are generally available and/or can provide useful information at the time that initial treatment decisions need to be made.

The value of bone marrow biopsy in this group of disorders is generally well established [1]. Bone marrow biopsy may help in confirming a suspected MDS by excluding reactive conditions in which dyshematopoietic changes may also be observed. Reactive conditions which may simulate MDS are listed in table 11. Among the most frequent causes of 'non-clonal' dyspoiesis encountered in clinical practice, it is worthwhile mentioning HIV-related myelodysplasia, autoimmune myelofibrosis, paraneoplastic myelodysplasia, and chronic parvovirus infection (fig. 6). All of these conditions are easily recognizable in the bone marrow biopsy (where most of them were initially described), but are not so easy to distinguish from MDS in the bone marrow aspirate smear. However, the need for clinical correlation to minimize the chances of 'overinterpretation' of the marrow findings cannot be overemphasized.

In MDS, bone marrow biopsy supplemented by immunohistochemistry can be used to increase the diagnostic accuracy and to refine the IPPS risk evaluation system $[14,15]$.

Bone marrow biopsy in MDS allows an accurate assessment of: marrow cellularity, predominant cell line(s), presence of fibrosis and/or other stromal changes, dysmegakaryopoiesis (which is more easily detected in histology preparations than in smears), and, finally, architectural disorganization. In normal marrow, granulopoietic precursors are mainly found in the paratrabecular region, while erythroid and megakaryocytes are more or less confined to the central marrow cavities. In MDS, topographical organization is lost, with precursors of different cell lines found in all marrow regions. Among the alterations detected by bone marrow biopsy, a prognostically important finding is the presence of aggregates or clusters of 'abnormally localized' immature myeloid pre- 
cursor cells (ALIP), i.e. myeloblasts and promyelocytes, in an abnormal central marrow cavity location (fig. 7a) $[16,17]$. Cases are classified as 'ALIP-positive' if at least three aggregates ( $>5$ myeloid precursors) or clusters (3-5 myeloid precursors) are identified in each section [17]. ALIP is mainly present in the aggressive MDS subtypes and is associated with a poor prognosis and an increased incidence of progression to acute leukemia. Presence of ALIP, however, is not unique to MDS and has been reported in reactive hematologic conditions (e.g. status post-bone marrow transplantation and post-induction chemotherapy) (table 12). In addition, the identification of the presence of ALIP may be compromised by using paraffin sections of excessive thickness or otherwise suboptimal morphology. CD34, an antigen expressed in progenitor and early precursor marrow cells, can be used as a 'surrogate marker' for the presence of ALIP (fig. 7b) [18, 19]. Both an increase in the percentage of CD34-positive cells and a tendency of positive cells to form aggregates have been shown to be reliable predictors of acute leukemic transformation and of poor survival in MDS cases, irrespective of their subtype $[13,19,20]$. This approach can be used to identify patients with MDS undergoing transition to AML, who are therefore candidates for early aggressive therapy. Most of the CD34-positive cells found in MDS morphologically resemble blasts. However, a proportion of the positive cells show promyelocytelike cytologic features. These should be counted as blasts rather than promyelocytes for the purpose of blast cell evaluation.

Aberrant expression of CD34 by megakaryocytes in MDSs has also been reported (fig. 8) [21]. In normal conditions, the CD34+ phenotype is only found on a small subset of megakaryocyte precursors morphologically identifiable as immature blasts. This suggests that the CD34-positive megakaryocytes seen in MDS represent poorly functional neoplastic megakaryocytes showing, in addition to morphologic atypia, anomalous phenotypic differentiation. However, CD34-positive megakaryocytes can also be identified in other types of neoplastic myeloid disorders, as well as in reactive conditions (e.g. megaloblastic anemia).

Immunohistology using CD34, CD42b, or CD61 may be useful in distinguishing MDS-f [22] (blasts CD34 positive and negative with CD42b, or CD61) from AMKL, a condition associated with an increased number of megakaryoblasts (positive with CD42 or CD61, and only variably positive with CD34) [6]. CD117 (c-Kit) has been recently proposed as an additional marker to identify myeloid precursor cells in marrow biopsies of patients with MDS. However, in our experience, this antibody is a less reliable marker than CD34, due to its weak and variable expression in MDS myeloblasts. Other antibodies which can be occasionally useful include: antihemoglobin [23] or glycophorin A to identify megaloblastic erythroblasts which can occasionally be confused with myeloid precursors (fig. 9); myeloperoxidase [24], which can be useful in differentiating background maturing myeloid precursors which are strongly myeloperoxidase positive from the leukemic myeloblasts which are characteristically weakly positive or negative in MDS cases, and CD68 and CD163 which can be used to identify background monocytes and in ruling out CMML [25, 26]. However, CMML marrows often show a predominance of granulocytes with only rare monocytes, with a characteristic discrepancy with the peripheral blood findings.

Immunohistologic analysis is especially helpful in three subsets of patients with MDS that may not have suitable marrow aspirate material for analysis: MDS-f $[22,27,28]$, therapy-related MDS (TR-MDS) $[10,29,30]$, and MDS with hypocellular marrow $[31,32]$. The presence of reticulin fibrosis and/or fatty changes in the bone marrow of these MDS patients, by causing hemodilution and poorly cellular smears, can make accurate disease characterization very difficult or impossible. The often low cellular yield of the bone marrow aspirate may also be insufficient to obtain adequate cytogenetic information, the importance of which has been discussed previously. Besides the presence of fibrosis, other common findings include the presence of areas of edema, increased number of microvessels which can be demonstrated also by immunostaining with vWF or CD34, frequent plasmacytosis, increased mast cells, macrophages with increased cellular debris and hemosiderin, lymphocytosis and lymphoid follicles. Loss of p53 function has a major role in the transformation process in hematologic malignancy. p53 alterations are also frequent in aggressive MDS, particularly in therapy-related cases, and in secondary AML $[30,33]$. A proportion of these cases have abnormalities of chromosome band 17p, site of the TP53 gene. The p53 gene product can be easily demonstrated in routinely processed bone marrow specimens (fig. 10). In our experience, p53 overexpression is almost always associated with the presence of a complex karyotypes and poor prognosis [30]. Therefore, p53 analysis of paraffin sections in MPD and MDS cases can be proposed as a 'surrogate marker' for cytogenetics when the latter approach is not available. 
8

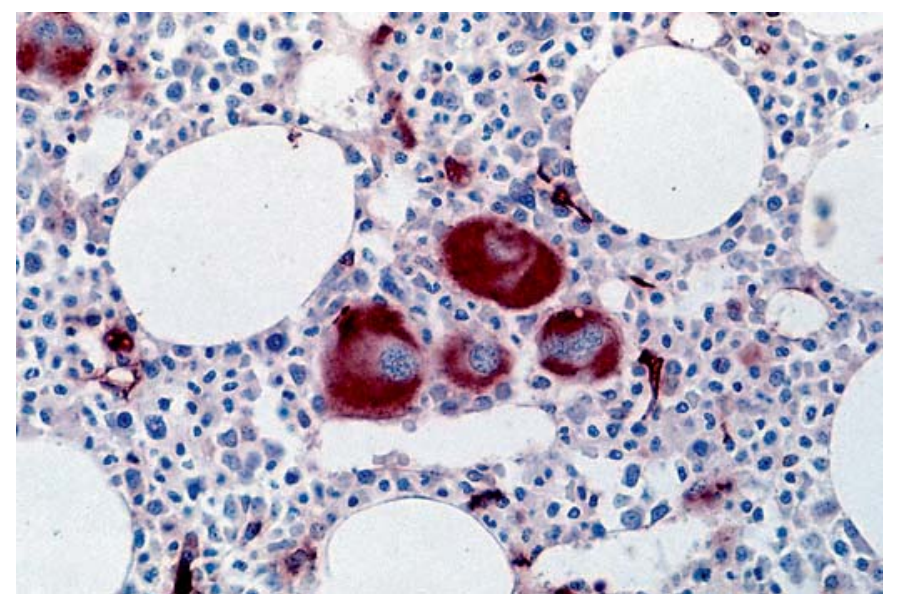

9

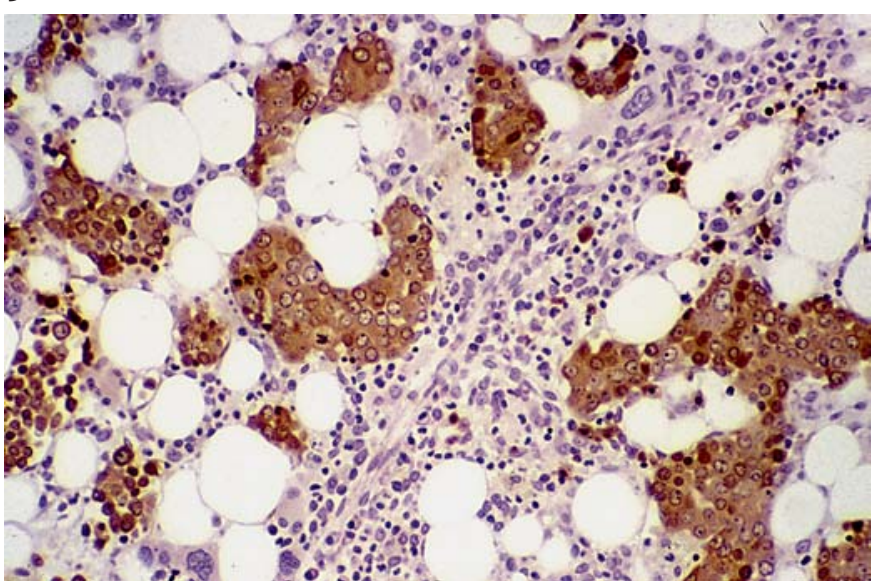

Fig. 8. MDS. The presence of aberrant expression of CD34 in megakaryocytes is a characteristic although non-specific finding in cases of MDS.

Fig. 9. Antihemoglobin immunostain is helpful in confirming the erythroblastic nature of clustered megaloblastic and/or dysplastic erythroblasts.

Fig. 10. Therapy-related MDS/AML. Overexpression of p53 protein is frequently seen in cases of therapy-related (alkylating) MDS or AML.

Fig. 11. Hypoplastic MDS. The main difficulty is to separate cases such as this one from cases of aplastic anemia. Immunohistology may be helpful (see fig. 5b).

10

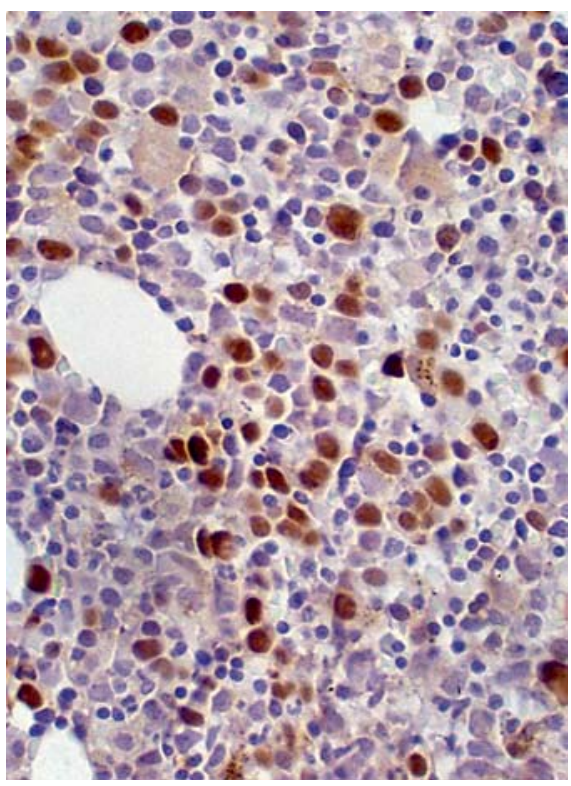

11

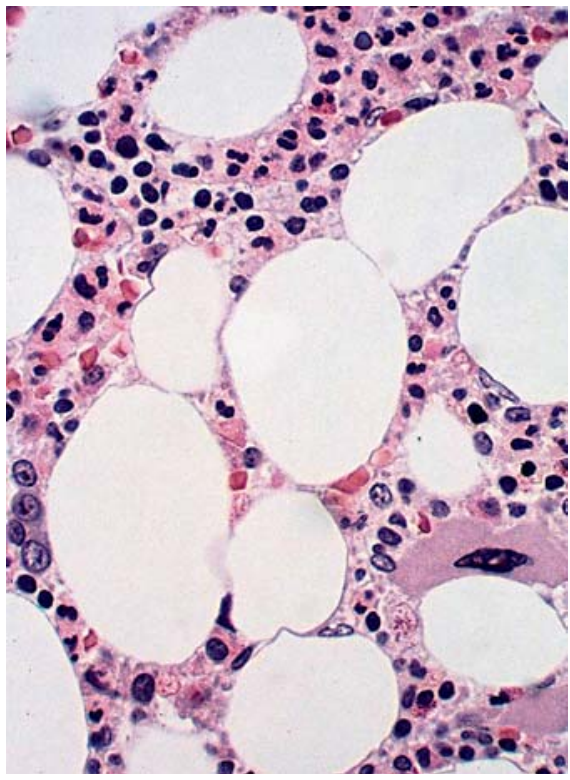

It is now well established that angiogenesis is involved in the pathogenesis of MDS. By immunostaining bone marrow biopsies with CD34 or other endothelium-reactive markers, such as CD105, CD31, vWF microvessel density has also been found to be increased in various leukemic disorders $[34,35]$. Results obtained in cases of MDSs suggest a correlation between increased angiogenesis, aggressive MDS subtypes, and rate of progression to acute leukemia [36].

\section{Myelodysplastic Syndromes with Fibrosis}

MDS- $\mathrm{f}$ is a MDS variant which is characterized by a marked increase in bone marrow reticulin fibers and presents with pancytopenia and minimal or absent organomegaly $[22,27,28]$ (table 13). The marrow shows trilineage dysplasia with prominent dysmegakaryopoiesis. In most cases an increased number of blasts are seen. The blasts are more easily documented in the marrow biopsy than in the aspirate, the latter being frequently suboptimal due to the presence of myelofibrosis. To qualify a case as MDS-f, a silver-stained reticulin fibrosis score of $\geq 2$ 
on the basis of the system proposed by Manoharan et al. [37] should be obtained.

MDS-f accounts for $10-15 \%$ of primary MDS cases and $>50 \%$ of TR-MDS. Within the subgroup of MDS-f, the classification according to FAB criteria reveals a majority of patients with RAEB. Only rare cases of RA with fibrosis have been reported. These rare cases seem to share the poor prognosis associated with the RAEB with fibrosis subtypes.

In MDS-f, the presence of increased CD34 expression in the marrow is often observed and the marker can be used to assess the blast count. The use of antibodies reactive with megakaryocytes has shown a higher number of these cells in MDS-f cases than either normal subjects or patients affected by MDS without fibrosis [22]. The megakaryocytes can show a particularly marked degree of pleomorphism with both small dwarf forms and large abnormal cells. The differential diagnosis of MDS-f includes several myelofibrotic myeloid neoplasms outlined in table 14. APMF is distinct from MDS-f only by its abrupt onset with fever and bone pain. In APMF, the histology of the marrow shows marked fibrosis ( $\geq 3$ according to the Manoharan grading system [37]) associated with numerous dwarf megakaryocytes, an increased number of blasts and severe trilineage dysplasia. Cases of RAEB-2 with fibrosis, except for the usually less acute clinical presentation, may be totally indistinguishable from APMF and probably represent the same disorder [6, 7]. The differential diagnosis includes also acute megakaryocytic leukemia which can morphologically overlap with APMF and AML-MLD. Chronic idiopathic myelofibrosis is usually easily distinguished by its characteristic morphologic features (e.g. intravascular hematopoiesis, giant megakaryocytes) and the presence of significant splenomegaly.

Primary MDS-f patients show an unfavorable prognosis mainly attributable to complications deriving from pancytopenia and continuous transfusions, with a life expectancy of 9.6 months, compared with 17.4 months in MDS without fibrosis [27].

\section{Therapy-Related Myelodysplastic Syndrome}

TR-MDS cases are usually clinically very aggressive diseases which should be considered as a separate type of MDS [38-40]. Two main types of TR-MDSs have been described. The first classical type typically occurs late, usually $\geq 7$ years after use of alkylating agents and presents as MDS with $-7 /$ del $7 \mathrm{q}$ and/or -5/del5q $[10,29,40]$.
Table 14. MDS-F and its separation from other myelofibrotic myeloid neoplasms

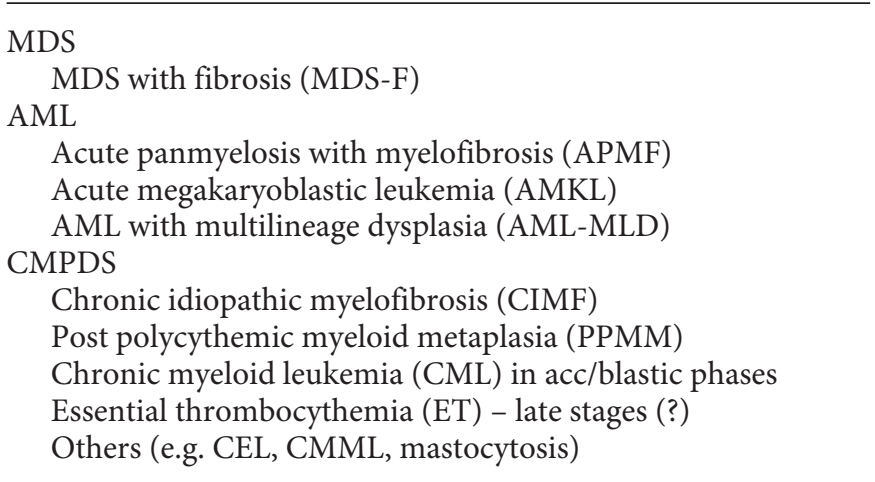

Table 15. MDS, hypoplastic

Cellularity $<30 \%$ (adjusted for age if $>65$ )

$5-15 \%$ of MDS

$\mathrm{F}>\mathrm{M}$; same age as other MDS

Most are RA

Cytopenia more severe

Role for immunosuppressive therapy

By the FAB system most cases fall within the RAEB category [10]. The second form occurs relatively early, in 2 3 years after the use of agents targeted at topoisomerase II (epipodophyllotoxins, e.g. etoposide, teniposide, doxorubicin), and presents with chromosomal translocations involving bands 11q23 and 21q22, or less frequently, other translocation which are more typical of the de novo leukemias [41]. Many of these patients progress directly to AML without a previously documented dysplastic phase. TR-MDSs with $17 \mathrm{p}$ deletions have morphologic features similar to those described before for the de novo cases with the same cytogenetic abnormality and are similarly characterized by the presence of p 53 mutations $[42,43]$.

The clinical features of TR-MDS are similar to those seen in aggressive primary MDS cases except for the more pronounced pancytopenia and anisopoikilocytosis which are usually associated with the former group. Occasional nucleated red blood cells are seen in the peripheral blood. The bone marrow is, on average, less cellular than in the primary cases, and significant reticulin fibrosis $(\geq 2+)$ is also more common. In spite of the similar degrees of fibrosis in both primary MDS-f and TR-MDS, the latter 
Table 16. Atypical CML

Atypical CML is a Ph'n-negative, $B C R / A B L$-negative myeloid disorder

Its incidence is $<2$ cases for 100 cases of $\mathrm{t}(9 ; 22)$, $B C R / A B L$-positive CML

Patients have some features of CML, e.g. splenomegaly, neutrophilia and anemia

No basophilia

Major characteristics to separate aCML from CML: dysgranulopoiesis which is often severe

Moreover, atypical CML may have an initial presentation more typical of MDS with anemia and normal to decreased PLT counts

Table 17. Chronic myelomonocytic leukemia

\begin{tabular}{ll}
\hline Peripheral blood & Bone marrow \\
\hline Persistent monocytosis of & Hypercellular \\
$>1 \times 10^{9} / 1$ with a percentage & Most cases show a \\
of monocytes $>10 \%$ of WBC & 'myeloproliferative look' \\
& High M:E ratio due to \\
& myeloproliferation \\
& Blast count is usually $<5 \%$ \\
& Variable fibrosis \\
\hline
\end{tabular}

group differs in terms of the number of megakaryoblasts and megakaryocytes which are significantly higher in the primary forms [22]. CD34 expression is almost always increased in this aggressive MDS subtype [10, 22]. In addition, p53 protein overexpression can be frequently observed, particularly in cases associated with prior alkylating agent chemotherapy (fig. 10) [30]. p53 expression was found to be associated with increased apoptosis in marrow hematopoietic cells and severe ineffective hematopoiesis [33].

\section{Hypoplastic Myelodysplastic Syndrome}

Hypoplastic MDS (h-MDS) accounts for about 15\% of the MDS cases, is more frequent in women, and occurs with an age-related frequency which is similar to that seen in primary MDS [32] (table 15). Previous genotoxic exposure or therapy needs to be excluded since hypocellular marrows can be seen in cases of TR-MDS. h-MDS is generally associated with pronounced cytope- nia, a finding which may suggest a diagnosis of acquired aplastic anemia. Bone marrow biopsy is necessary to diagnose this variant. Most investigators consider a case of MDS as hypocellular when the marrow cellularity is $<30 \%$. However, correction for age is recommended as a value of $20 \%$ may be still within normal range for patients $>60$ years of age [44]. Within the subgroup of MDS with hypoplastic bone marrow, classification according to FAB criteria reveals a majority of patients with RA ( $66.7 \%$ in one series). The presence of $\geq 20 \%$ myeloblasts in a hypocellular marrow rules out MDS in favor of hypocellular AML. Dysplastic features in hematopoietic cells occur less frequently and are of lower grade in comparison to normo- to hypocellular MDS. In the author's experience, most h-MDS patients with RA have only mild dyserythropoiesis. Since these patients do not have an increased proportion of blasts in the aspirate or ALIP in the bone marrow biopsy, the separation from aplastic anemia may be problematic (fig. 11). This is also compounded by the high proportion of cases showing a mesenchymal reaction, especially an increase of mast cells, and reactive lymphoid follicles, features similar to those observed in bone marrow biopsies obtained from patients with aplastic anemia. The presence of easily identifiable megakaryocytes within an architecturally disorganized marrow and the presence of reticulin fibrosis favor MDS over aplastic anemia. Immunohistochemistry can help in distinguishing h-MDS from acquired aplastic anemia, the former disorder being characterized by higher CD34 expression as compared to aplastic anemia [31, 45]. Finally, bone marrow cellularity does not appear to be an important prognostic factor in MDS, because patients with hypocellular MDS have a similar prognosis to those cases of MDS with normo/hypercellular marrows [46]. The distinction from aplastic anemia, however, is significant because the risk of progression to acute leukemia is much greater in h-MDS [47].

\section{Myelodysplastic/Myeloproliferative Disorders}

The category of MDS/MPD disorders includes malignant hematopoietic proliferations which, at the time of their initial presentation, display features of both MDSs and MPDs [48-50]. Cytopenias and dysplastic changes of any cell line may be seen, similar to the MDSs. Elevated white blood cell (WBC) counts, hypercellular marrows with fibrosis, and organomegaly, features more commonly associated with MPDs, may also be present. The pres- 
ence of fibrosis alone in cases that are otherwise typical of myelodysplasia should not justify placement in this category. The three best-defined mixed myeloproliferative and myelodysplastic syndromes are: aCML, CMML, and juvenile myelomonocytic leukemia.

\section{Atypical Chronic Myeloid Leukemia}

aCML [48] is a Philadelphia chromosome-negative and $B C R / A B L$-negative proliferative disorder that affects elderly patients with an apparent male predominance. Its incidence is $<2$ cases for every 100 cases of $\mathrm{t}(9 ; 22), B C R /$ $A B L$-positive CML [50] (table 16). Patients have some features of CML including: splenomegaly, an elevated WBC count of predominantly granulocytic cells, and moderate anemia. The major characteristic which distinguishes atypical CML is dysgranulopoiesis, which is often severe. Occasionally, atypical CML may have an initial presentation more typical of myelodysplasia with a low WBC count and normal to decreased platelet counts [49]. The WBCs are shifted to the left with immature granulocytes, including blast cells, promyelocytes, and myelocytes, representing $10-20 \%$ of peripheral blood white cells. Dysplastic neutrophils are typically seen. Granulocytes may show typical pseudo-Pelger-Huët changes, 'mononuclear' cell-like morphology due to nuclear condensation, and cytoplasmic hypogranularity. Monocytes are usually $<10 \%$ of peripheral blood cells. In contrast to CML, basophilia is not prominent, usually accounting $<2 \%$ of peripheral blood white cells.

The bone marrow is hypercellular, with an elevated myeloid-to-erythroid ratio, and marrow fibrosis may be present (fig. 12a). The bone marrow biopsy demonstrates granulocytic hyperplasia with an increased proportion of blasts $(<20 \%)$. These cells can be highlighted by immunostaining with CD34. Dysmegakaryopoiesis is easily identified. A predominance of small megakaryocytic forms, which can be similar in appearance to those seen in MDS, may be observed. The myeloid-to-erythroid ratio is usually less than $10: 1$, and there is no evidence of the Philadelphia chromosome by either routine karyotype or molecular studies for the $B C R / A B L$ fusion product. Although some abnormalities of granulocyte nuclear lobation may be seen in CML, particularly in the accelerated phase, atypical CML commonly has more typical dysplastic changes that may involve all cell lines (trilineage dysplasia). In addition to dysgranulopoiesis, dyserythropoiesis and megakaryocyte dysplasia (fig. 12b) are common, and megakaryocytes may be reduced in number with associated thrombocytopenia. Atypical CML is a more aggressive disease than $\mathrm{CML}$, and pro-

Histopathology in the Diagnosis and Classification of Myeloid Neoplasms gression usually occurs within 2 years $[49,51]$. Patients may develop acute leukemia or may have bone marrow failure secondary to marked fibrosis.

Cytogenetic and molecular genetic studies are essential in the diagnosis of atypical CML to exclude $t(9 ; 22)$ or the $B C R / A B L$ fusion product of usual-type CML. There is no known defining cytogenetic abnormality for atypical CML, but del(20q11) and trisomy 8 have been reported [50]. Other ancillary studies, particularly immunophenotyping studies, do not usually help unless blast cell numbers are elevated.

\section{Chronic Myelomonocytic Leukemia}

CMML has features both of a myeloproliferative and a myelodysplastic syndrome. The disease has been divided into myelodysplastic and myeloproliferative subtypes on the basis of a WBC count of $13 \times 10^{9} / 1$ or higher for the myeloproliferative type and less than that number for the myelodysplastic type. Patients with leukocytosis of $>13 \times 10^{9} /$ have a higher incidence of splenomegaly [48]. However, both subtypes show a similar degree of dysplastic changes. Since the percentage of bone marrow (or peripheral blood) blasts is the most important determinant of survival in CMML patients, the use of a WBC cutoff to separate the subtypes is probably arbitrary and of controversial prognostic value [52].

The diagnosis (table 17) requires a persistent peripheral blood monocytosis of $>1 \times 10^{9} / 1$, with a percentage of monocytes $>10 \%$ of the WBCs. The monocytes may be abnormal in appearance with bizarre nuclei and even cytoplasmic granules. Promonocytes, with more immature nuclear chromatin, may be present in the blood, but monoblasts are usually not present or represent $<2 \%$ of peripheral blood cells. If blasts and promonocytes account for $\geq 20 \%$ of the WBCs, the diagnosis should be AML rather than CMML. The peripheral blood may demonstrate dysplastic changes typical of the MDSs, or dysplastic changes may be minimal. Other changes in the peripheral blood are variable, however mild anemia is usually seen. Although an elevated peripheral blood monocyte count is necessary for the diagnosis of CMML, such a diagnosis should never be made without examination of the bone marrow. Some AMLs with monocytic blasts may show peripheral blood changes similar to those of CMML because of cytologic maturation of the blast cell population in the peripheral blood. The bone marrow of CMML is usually hypercellular and may demonstrate monocytic or granulocytic hyperplasia (fig. 13a). When granulocytic hyperplasia is prominent, it may be difficult to distinguish the abnormal monocyte population from myelocytes. Non- 

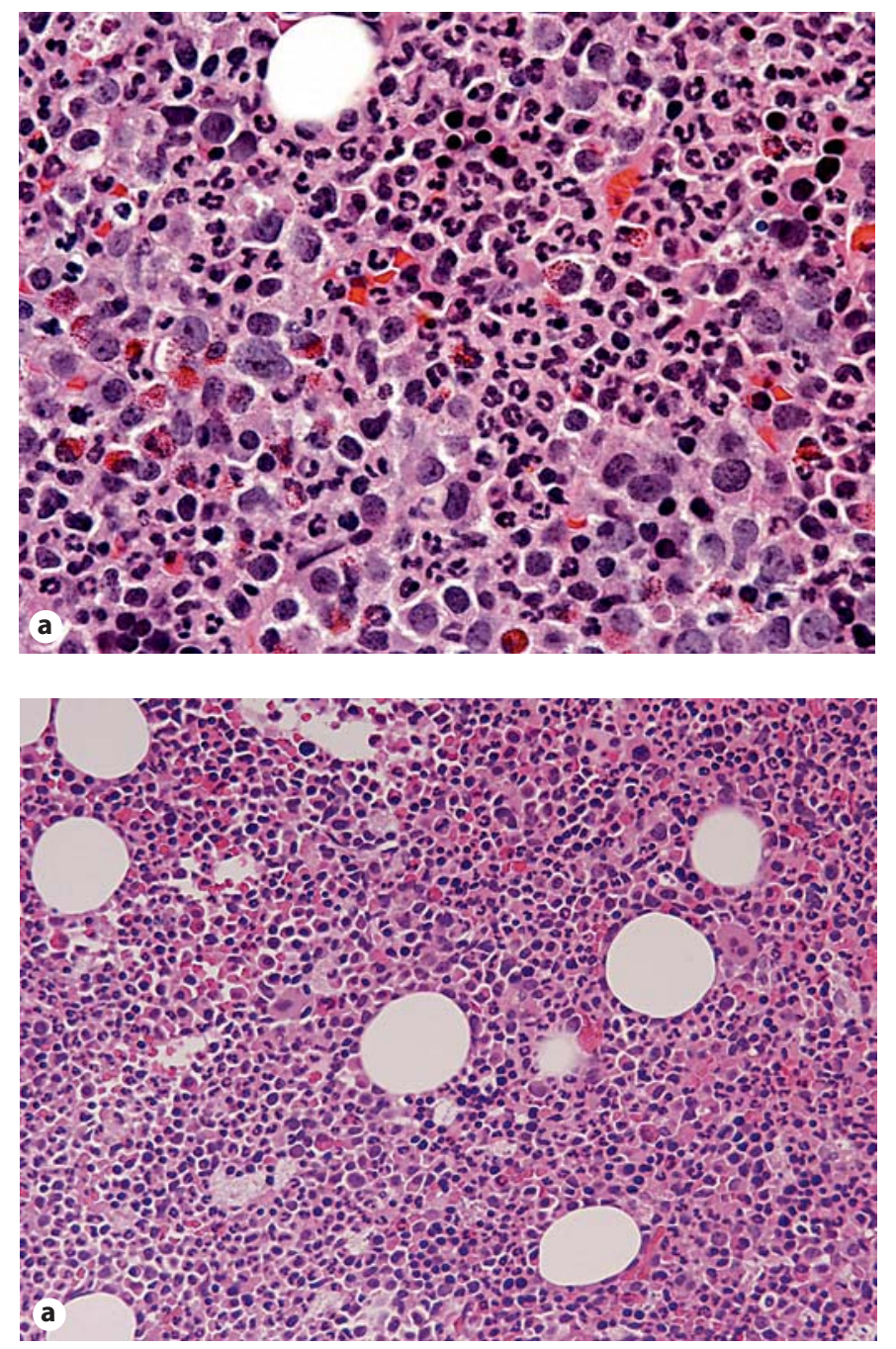

Fig. 12. MDS/MPD. a Atypical CML. Note the predominant granulocytic proliferation (MPD-like) associated with an increased number of blasts. b CD42 may help in identified dysmegakaryopoietic forms frequently found in cases of MDS/MPD.

Fig. 13. CMML. a The striking myeloproliferative appearance makes it difficult to distinguish this case of CMML from CML or aCML. b CD68R immunostaining identifies a few monocytes but is not as sensitive as NBE (as shown in fig. 14).

Fig. 14. CMML. Naphthyl butyrate esterase facilitates the identification of monocytes and promonocytes in marrow aspirate of cases of CMML.
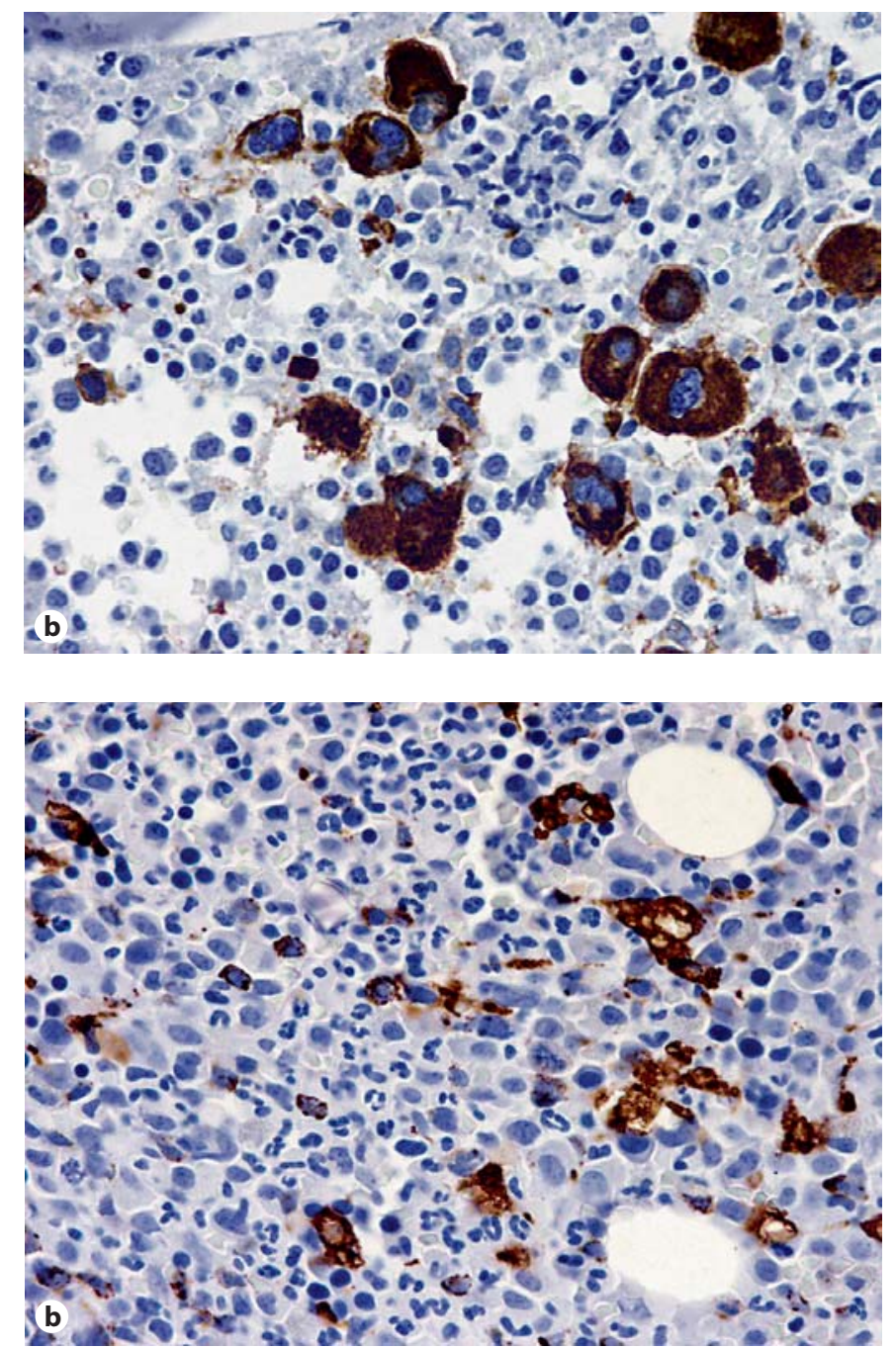
specific esterase cytochemical studies may be helpful in this setting, by highlighting the abnormal monocytes (fig. 14) [26]. Flow cytometry with CD14, CD11c, CD64, and immunohistochemistry with CD68 (KP-1), CD163, and CD68R (PG-M1) are less useful in confirming the presence of monocytic differentiation than cytochemistry (fig. 13b) [26]. Erythroid precursors and megakaryocytes may demonstrate prominent dysplastic changes, but these cell types are often normal in appearance. Ringed sideroblasts are present in increased numbers in some cases. Blast cell and promonocyte counts may be elevated up to $20 \%$, and an elevation in blast cell numbers is associated with a poorer prognosis $[53,54]$. The WHO classification scheme subdivides CMML into two subcategories, depending on the number of blasts found in the peripheral blood and bone marrow. These include: CMML-1 with blasts $<5 \%$ in the blood or $<10 \%$ in the bone marrow, and CMML-2 which is characterized by blasts $5-19 \%$ in the blood or $10-19 \%$ in the bone marrow, or when Auer rods are present and the blast count is $<20 \%$ in blood or marrow. The finding of $\geq 20 \%$ blasts in the blood or the bone marrow indicate AML rather than CMML.

Cytogenetic and molecular genetic studies, particularly the absence of the Philadelphia chromosome and $B C R / A B L$, help in excluding CML. An additional subset, CMML with eosinophilia, may be diagnosed when the criteria for CMML are present, but in addition, the eosinophil count in the peripheral blood is $>1.5 \times 10^{9} / 1$. Patients in this category may have symptoms related to the degranulation of the eosinophils comparable to those seen in hypereosinophilic syndrome. This subset should be designated as CMML-1 or CMML-2 with eosinophilia according to the above guidelines. Mutations of RAS are detected in approximately one third of CMML cases. Although detection of RAS mutations is not generally useful for diagnosis, abnormalities involving the RAS pathway are thought to be an important mechanism for this and other CMPDs, including CML and cases associated with $\mathrm{t}(5 ; 12)$ chromosome abnormality [55].

The differential diagnosis between atypical CML and CMML may be difficult, but is critical because of the worse prognosis of patients with atypical CML compared with CMML. CMML may be distinguished from atypical CML by peripheral blood features $[26,48]$, but some overlap with atypical CML may occur. Although monocyte counts may be slightly elevated in atypical CML, these cells do not usually exceed $10 \%$, whereas monocyte counts in CMML are usually $>10 \%$. The degree of granulocyte dysplasia in CMML is also not as pronounced as is usually seen in atypical CML. Atypical CML demon-

Histopathology in the Diagnosis and Classification of Myeloid Neoplasms strates an increase in immature granulocytic cells, including blast cells, promyelocytes, and myelocytes, of up to $20 \%$ in the peripheral blood; these cell types are almost always $<10 \%$ in the blood of patients with CMML.

Juvenile myelomonocytic leukemia, a member of the WHO MDS/MPD diseases group, is currently included within the group of pediatric MDSs [56] and is therefore not included in this review.

\section{Other Myelodysplastic/Myeloproliferative Syndromes}

Some cases demonstrate features of both myelodysplasia and myeloproliferative syndromes and do not fit well into any of the previously mentioned categories. Many of these cases have typical features of myelodysplasia as well as an atypical finding more suggestive of a MPD, such as marked marrow fibrosis, leukocytosis, or organomegaly. Such cases may be termed mixed myeloproliferative/myelodysplastic syndromes, not further classifiable, with a comment describing the atypical findings. One such syndrome has features of RA with ringed sideroblasts and thrombocythemia (acquired sideroblastic anemia associated with thrombocytosis) [57-58]. These cases have no sex predilection or specific cytogenetic abnormality and must be differentiated from the 5q- syndrome myelodysplasias. A mixed MDS/MPD disorder associated with isochromosome $17 \mathrm{q}$ has been described; it occurs with a male predominance in adults and is associated with severe hyposegmentation of neutrophil nuclei, monocytosis, and a high rate of transformation to AML. Poorly characterized MDS/MPD neoplasms may also occur in association with mast cell disease where they represents one of the types of clonal non-mast cell lineage hematological diseases associated with systemic mastocytosis [59].

This review article stresses the need for integration between morphology, immunophenotype, genetic features, and clinical manifestations in diagnosing myeloid neoplasms. In this article, although much emphasis has been placed on the histologic interpretation of the bone marrow biopsy, the need for a comprehensive evaluation of marrow samples cannot be overemphasized. This multiparametric approach forms the basis for the WHO classification of tumors of hematopoietic and lymphoid tissues. Only by following this principle can the hematopathologist reliably and reproducibly identify rare variants of myeloid neoplasms such chronic myelomonocytic leukemia, atypical chronic myeloid leukemia, fibrotic and/or hypoplastic subtypes of myelodysplastic syn- 
dromes and of acute myeloid leukemia. The latter two disease groups include the important subsets of patients with therapy-related or secondary (to previous MDS, MDS/MPD, or MPD) myeloid neoplasms. Since all these entities have, usually, some degree of associated myelofibrosis, bone marrow biopsy often represents the only game in town. When supplemented by immunohistology it is an essential tool for assessing the frequency of marrow blasts and their lineage derivation. Novel cytogenetics and molecular genetic approaches are likely to revolutionize the way we classify these diseases in the near future. However, it is highly unlikely that these new techniques will be capable, on their own, of adequately stratifying patients for purpose of treatment.

The bone marrow biopsy has a bright future ahead. It represents the only in situ approach that allows the identification of specific cell subsets within their unique spatial relationships. This is of course particularly valuable in analyzing neoplastic populations present within highly polymorphic cellular backgrounds, such as those normally seen in bone marrow. New approaches will further enhance its value. Several recent studies have demon- strated the usefulness of microdissection techniques in the demonstration of important biologic characteristics of selected malignant cells present within a mixed bone marrow cellularity. Examples of these applications have included the detection of additional genetic events in microdissected megakaryocytes, the demonstration of clonality in low-grade myelodysplastic syndromes, and the objective confirmation of lymphomatous involvement in bone marrows containing lymphoid aggregates in patients with lymphoproliferative disorders. The possibility of analyzing, by genetic techniques, clusters of blasts microdissected from biopsies will allow a better demonstration of disease progression and of clonal evolution, in cases in which the blast count on the bone marrow aspirate is still 'falsely' low. This will open the possibility of better assessing post-transplant or post-chemotherapy marrow samples by accurately distinguishing between early regeneration, cytokine effects, and residual leukemic disorder. Even with the most sophisticated technology at one's disposal, an adequate characterization of bone marrow morphology will represent the best 'reality check' available to us for many years to come.

\section{References}

1 Orazi A, O'Malley DP, Arber D: Illustrated Pathology of the Bone Marrow. New York, Cambridge University Press, 2006.

-2 Bennett JM, Catovsky D, Daniel MT, Flandrin G, Galton DA, Gralnick HR, Sultan C: Proposals for the classification of the acute leukemias. Br J Haematol 1976;33:451-458.

-3 Lu G, Altman AJ, Benn PA: Review of the cytogenetic changes in acute megakaryoblastic leukemia: one disease or several? Cancer Genet Cytogenet 1993;67:81-89.

-4 Lion T, Haas OA, Harbott J, Bannier E, Ritterbach J, Jankovic M, Fink FM, Stojimirovic A, Herrmann J, Riehm HJ: The translocation $\mathrm{t}(1 ; 22)(\mathrm{p} 13 ; \mathrm{q} 13)$ is a nonrandom marker specifically associated with acute megakaryocytic leukemia in young children. Blood 1992;79:3325-3330.

5 Orazi A, Neiman RS, Ulbright TM, Heerema NA, John K, Nichols CR: Hematopoietic precursor cells within the yolk sac tumor component are the source of secondary hematopoietic malignancies in patients with mediastinal germ cell tumors. Cancer 1993;71:3873-3881.

6 Orazi A, O’Malley DP, Jiang J, Vance GH, Thomas J, Czader MB, Fang W, An C, Banks PM: Acute panmyelosis with myelofibrosis: an entity distinct from acute megakaryoblastic leukemia. Mod Pathol 2005; 18:603-614.
7 Thiele J, Kvasnicka HM, Zerhusen G, Vardiman J, Diehl V, Luebbert M, Schmitt-Graeff A: Acute panmyelosis with myelofibrosis: a clinicopathological study on 46 patients including histochemistry of bone marrow biopsies and follow-up. Ann Hematol 2004; 83:513-521.

$>8$ Gahn B, Haase D, Unterhalt M, Drescher M, Schoch C, Fonatsch C, Terstappen LW, Hiddemann W, Buchner T, Bennett JM, Wormann B: De novo AML with dysplastic hematopoiesis: cytogenetic and prognostic significance. Leukemia 1996;10:946-951.

9 Brunning RD, Matutes E, Harris NL, Flandrin G, Vardiman J, Bennett J, Head D: Acute myeloid leukaemia with multilineage dysplasia; in Jaffe ES, Harris NL, Stein H, Vardiman JW (eds): World Health Organization Classification of Tumors: Pathology and Genetics of Tumours of Haematopoietic and Lymphoid Tissues. Lyon, IARC Press, 2001, pp 88-89.

-10 Orazi A, Cattoretti G, Soligo D, Luksch R, Lambertenghi-Deliliers G: Therapy-related myelodysplastic syndromes: FAB classification, bone marrow histology, and immunohistology in the prognostic assessment. Leukemia 1993;7:838-847.
11 Arber DA, Stein AS, Carter NH, Ikle D, Forman SJ, Slovak MLL: Prognostic impact of acute myeloid leukemia classification. Importance of detection of recurring cytogenetic abnormalities and multilineage dysplasia on survival. Am J Clin Pathol 2003;119:672-680.

12 Pedersen-Bjergaard J, Andersen MK, Christiansen DH: Therapy-related acute myeloid leukemia and myelodysplasia after highdose chemotherapy and autologous stem cell transplantation. Blood 2000;95:32733279.

13 Rowley JD, Olney HJ: International Workshop on the Relationship of Prior Therapy to Balanced Chromosome Aberrations in Therapy-Related Myelodysplastic Syndromes and Acute Leukemia: Overview report. Genes Chromosomes Cancer 2002;33:331-345.

14 Lambertenghi-Deliliers G, Annaloro C, Soligo D, Oriani A: The diagnostic and prognostic value of bone marrow immunostaining in myelodysplastic syndromes. Leuk Lymphoma 1998;28:231-239.

15 Verburgh E, Achten R, Maes B, Hagemeijer A, Boogaerts M, De Wolf-Peeters C, Verhoef G: Additional prognostic value of bone marrow histology in patients subclassified according to the International Prognostic Scoring System for myelodysplastic syndromes. J Clin Oncol 2003;21:273-282. 
-16 Tricot G, De Wolf-Peeters C, Vlietinck R, Verwilghen RL: Bone marrow histology in myelodysplastic syndromes. II. Prognostic value of abnormal localization of immature precursors in MDS. Br J Haematol 1984;58: 217-225.

17 De Wolf-Peeters C, Stessens R, Desmet V, Tricot G, Verwilghen RL: The histological characterization of ALIP in the myelodysplastic syndromes. Pathol Res Pract 1986; 181:402-407.

18 Orazi A: CD34 immunoperoxidase staining for the diagnosis of myelodysplastic syndromes and chronic myeloid leukaemia. J Clin Pathol 1995;48:884.

-19 Soligo DA, Oriani A, Annaloro C, Cortelezzi A, Calori R, Pozzoli E, Nosella D, Orazi A, Deliliers GL: CD34 immunohistochemistry of bone marrow biopsies: prognostic significance in primary myelodysplastic syndromes. Am J Hematol 1994;46:9-17.

-20 Baur AS, Meuge-Moraw C, Schmidt PM, Parlier V, Jotterand M, Delacretaz F: CD34/ QBEND10 immunostaining in bone marrow biopsies: an additional parameter for the diagnosis and classification of myelodysplastic syndromes. Eur J Haematol 2000;64:71-79.

-21 Pellegrini W, Facchetti F, Marocolo D, Pelizzari AM, Capucci A, Rossi G: Expression of CD34 by megakaryocytes in myelodysplastic syndromes. Haematologica 2000; 85:1117-1118.

-22 Lambertenghi-Deliliers G, Orazi A, Luksch R, Annaloro C, Soligo D: Myelodysplastic syndrome with increased marrow fibrosis: a distinct clinico-pathological entity. Br J Haematol 1991;78:161-166.

-23 Neiman RS: Erythroblastic transformation in myeloproliferative disorders: confirmation by an immunohistologic technique. Cancer 1980;46:1636-1640.

24 Pinkus GS, Pinkus JL: Myeloperoxidase: a specific marker for myeloid cells in paraffin sections. Mod Pathol 1991;4:733-741.

-25 Manaloor EJ, Neiman RS, Heilman DK, Albitar M, Casey T, Vattuone T, Kotylo P, Orazi A: Immunohistochemistry can be used to subtype acute myeloid leukemia in routinely processed bone marrow biopsy specimens. Comparison with flow cytometry. Am J Clin Pathol 2000;113:814-822.

-26 Orazi A, Chiu R, O’Malley DP, Czader M, Allen SL, An C, Vance GH: Chronic myelomonocytic leukemia: the role of bone marrow biopsy immunohistology. Mod Pathol 2006;19:1536-1545.

-27 Maschek H, Georgii A, Kaloutski V, Werner M, Bandecar K, Kressel MG, Choritz H, Freund M, Hufnagl D: Myelofibrosis in primary myelodysplastic syndromes: a retrospective study of 352 patients. Eur J Haematol 1992;48:208-214.

Histopathology in the Diagnosis and Classification of Myeloid Neoplasms
28 Pagliuca A, Layton DM, Manoharan A, Gordon S, Green PJ, Mufti GJ: Myelofibrosis in primary myelodysplastic syndromes: a clinico-morphological study of 10 cases. Br J Haematol 1989;71:499-504.

29 Levine EG, Bloomfield CD: Leukemias and myelodysplastic syndromes secondary to drug, radiation, and environmental exposure. Semin Oncol 1992;19:47-84.

30 Orazi A, Cattoretti G, Heerema NA, Sozzi G, John K, Neiman RS: Frequent p53 overexpression in therapy-related myelodysplastic syndromes and acute myeloid leukemias: an immunohistochemical study of bone marrow biopsies. Mod Pathol 1993;6: 521-525.

31 Orazi A, Albitar M, Heerema NA, Haskins S, Neiman RS: Hypoplastic myelodysplastic syndromes can be distinguished from acquired aplastic anemia by CD34 and PCNA immunostaining of bone marrow biopsy specimens. Am J Clin Pathol 1997; 107:268-274.

32 Maschek H, Kaloutsi V, Rodriguez-Kaiser M, Werner M, Choritz H, Mainzer K, Dietzfelbinger M, Georgii A: Hypoplastic myelodysplastic syndrome: incidence, morphology, cytogenetics, and prognosis. Ann Hematol 1993;66:117-122.

33 Orazi A, Kahsai M, John K, Neiman RS: p53 overexpression in myeloid leukemic disorders is associated with increased apoptosis of hematopoietic marrow cells and ineffective hematopoiesis. Mod Pathol 1996;9:4852.

34 Ponzoni M, Savage DG, Ferreri AJM, Pruneri G, Viale G, Servida P, Bertolini F, Orazi A: Chronic idiopathic myelofibrosis: independent prognostic importance of bone marrow microvascular density evaluated by CD105 (endoglin) immunostaining. Mod Pathol 2004;17:1513-1520.

35 Molica S, Vacca A, Levato D, Merchionne F, Ribatti D: Angiogenesis in acute and chronic lymphoblastic leukemia. Leuk Res 2004; 28:321-324.

36 Pruneri G, Bertolini F, Soligo D, Carboni N, Cortelezzi A, Ferrucci PF, Buffa R, Lambertenghi-Deliliers G, Pezzella F: Angiogenesis in myelodysplastic syndromes. Br J Cancer 1999;81:1398-1401.

37 Manoharan A, Horsley R, Pitney WR: The reticulin content of bone marrow in acute leukaemia in adults. Br J Haematol 1979;43: 185-190.

- 38 Pedersen-Bjergaard J, Philip P, Larsen SO, Andersson M, Daugaard G, Ersboll J, Hansen SW, Hou-Jensen K, Nielsen D, Sigsgaard TC, et al: Therapy-related myelodysplasia and acute myeloid leukemia. Cytogenetic characteristics of 115 consecutive cases and risk in seven cohorts of patients treated intensively for malignant diseases in the Copenhagen series. Leukemia 1993;7:1975-1986.
39 Ellis M, Ravid M, Lishner M: A comparative analysis of alkylating agent and epipodophyllotoxin-related leukemias. Leuk Lymphoma 1993;11:9-13.

40 Michels SD, McKenna RW, Arthur DC, Brunning RD: Therapy-related acute myeloid leukemia and myelodysplastic syndrome: a clinical and morphologic study of 65 cases. Blood 1985;65:1364-1372.

41 Quesnel B, Kantarjian H, Bjergaard JP, Brault P, Estey E, Lai JL, Tilly H, Stoppa AM, Archimbaud E, Harousseau JL, et al: Therapy-related acute myeloid leukemia with $t(8 ; 21)$, inv $(16)$, and $t(8 ; 16)$ : a report on 25 cases and review of the literature. J Clin Oncol 1993;11:2370-2379.

42 Lai JL, Preudhomme C, Zandecki M, Flactif M, Vanrumbeke M, Lepelley P, Wattel E, Fenaux P: Myelodysplastic syndromes and acute myeloid leukemia with 17-p deletion. An entity characterized by specific dysgranulopoiesis and a high incidence of p53 mutations. Leukemia 1995;9:370-381.

43 Sugimoto K, Hirano N, Toyoshima H, Chiba S, Mano H, Takaku F, Yazaki Y, Hirai H: Mutations of the p53 gene in myelodysplastic syndrome (MDS) and MDS-derived leukemia. Blood 1993;81:3022-3026.

-44 Tuzuner N, Cox C, Rowe JM, Watrous D, Bennett JM: Hypocellular myelodysplastic syndromes: new proposals. Br J Haematol 1995;91:612-617.

45 Matsui WH, Brodsky RA, Smith BD, Borowitz MJ, Jones RJ: Quantitative analysis of bone marrow CD34 cells in aplastic anemia and hypoplastic myelodysplastic syndromes. Leukemia 2006;20:458-462.

46 Goyal R, Qawi H, Ali I, Dar S, Mundle S, Shetty V, Mativi Y, Allampallam K, Lisak L, Loew J, Venugopal P, Gezer S, Robin E, Rifkin S, Raza A: Biologic characteristics of patients with hypocellular myelodysplastic syndromes. Leuk Res 1999;23:357364.

47 Barrett J, Saunthararajah Y, Molldrem J: Myelodysplastic syndrome and aplastic anemia: distinct entities or diseases linked by a common pathophysiology? Semin $\mathrm{He}-$ matol 2000;37:15-29.

48 Bennett JM, Catovsky D, Daniel MT, Flandrin G, Galton DA, Gralnick H, Sultan C, Cox C: The chronic myeloid leukemias: guidelines for distinguishing chronic granulocytic, atypical chronic myeloid, and chronic myelomonocytic leukemia. Proposals by the French American-British Cooperative Leukemia Group. Br J Haematol 1994;87:746-754.

49 Oscier D: Atypical chronic myeloid leukemias. Pathol Biol (Paris) 1997;45:587-593. 
50 Vardiman, JW, Imbert M, Pierre R, Bain B, Brunning RD, Flandrin G: Atypical chronic myeloid leukaemia; in Jaffe ES, Harris NL, Stein H, Vardiman JW (eds): World Health Organization Classification of Tumors: Pathology and Genetics of Tumours of Haematopoietic and Lymphoid Tissues. Lyon, IARC Press, 2001, pp 53-54.

51 Martiat P, Michaux JL, Rodhain J: Philadelphia-negative $(\mathrm{Ph}-)$ chronic myeloid leukemia (CML): comparison with $\mathrm{Ph}+\mathrm{CML}$ and chronic myelomonocytic leukemia. Blood 1991;78:205-211.

- 52 Germing U, Gattermann N, Minning H, Heyll A, Aul C: Problems in the classification of CMML-dysplastic versus proliferative type. Leuk Res 1998;22:871-878.
3 Fenaux P, Beuscart R, Lai JL, Jouet JP, Bauters F: Prognostic factors in adult chronic myelomonocytic leukemia: an analysis of 107 cases. J Clin Oncol 1988;6: 1417-1424.

54 Storniolo AM, Moloney WC, Rosenthal DS, Cox C, Bennett JM: Chronic myelomonocytic leukemia. Leukemia 1990;4:766-770.

55 Steer EJ, Cross NC: Myeloproliferative disorders with translocations of chromosome 5q31-35; role of the platelet-derived growth factor receptor $\beta$. Acta Haematol 2002;107: 113-122.
Niemeyer CM, Kratz CP, Hasle H: Pediatric myelodysplastic syndromes. Curr Treat Options Oncol 2005;6:209-214.

57 Shaw GR: Ringed sideroblasts with thrombocytosis: an uncommon mixed myelodysplastic/myeloproliferative disease of older adults. Br J Haematol 2005;131:180-184.

58 Schmitt-Graeff A, Thiele J, Zuk I, Kvasnicka HM: Essential thrombocythemia with ringed sideroblasts: a heterogeneous spectrum of diseases, but not a distinct entity. Haematologica 2002;87:392-399.

59 Horny HP, Sotlar K, Sperr WR, Valent P: Systemic mastocytosis with associated clonal haematological non-mast cell lineage diseases: a histopathological challenge. J Clin Pathol 2004;57:604-608. 